\title{
ARTí́CULO
}

\section{Evaluación del desempeño de la primera reserva marina de Chile, La Rinconada, en el ámbito de la conservación de su especie focal, el ostión del norte Argopecten purpuratus}

Evaluation of the performance of the first marine reserve in Chile, La Rinconada, in the field of conservation on its focal species, the scallop Argopecten purpuratus

\section{Miguel Avendaño ${ }^{1 *}$ y Marcela Cantillánez ${ }^{1}$}

\begin{abstract}
${ }^{1}$ Departamento de Ciencias Acuáticas y Ambientales, Universidad de Antofagasta, Angamos 601, Antofagasta, Chile. *miguel.avendano@uantof.cl

Abstract.- We evaluated the performance of the first marine reserve in Chile, created in La Rinconada, Antofagasta, based on biotic indicators (abundance; population structure and recruitment success) of its focal species the scallop Argopecten purpuratus. This was attained by analyzing species population information, acquired during 2001, 2002, 2005 and 2009, by direct assessment diving, and on spat settlement into artificial collectors. The results show variation in the density of the specimens, which strongly affects the total abundance of the population, with a significant decrease in their average size in time, which is a decrease of individuals on the minimum legal size $(90 \mathrm{~mm})$ from $2.6 \times 10^{6}$ individuals in 2001 to $1.5 \times 10^{5}$ individual in 2009. This reduction has generated a drastic decline in their spawning stock, which has seriously impacted on the uptake of seed, descending from 15,340 ind. collector ${ }^{-1}$ in 2001 to 1,157 ind. collector ${ }^{-1}$ in 2009. The analysis of these results through 3 indicators, indicates that the measures of conservation have failed to recover its density and size structure, to enhance the availability of seeds and breeding adults, which were the main purposes for the creation of this marine reserve. While the main reason that inspired its creation was the conservation of the resource A. purpuratus, this measure has resulted in increased interest for illegal exploitation. The need for implementing measures that allow effective participation is concluded, with equity regarding resource utilization, considering that after 20 years of fishing ban, regulatory measures imposed by the State, have not had the expected results.
\end{abstract}

Key words: Performance, Marine Reserve, Argopecten purpuratus, Chile

\begin{abstract}
Resumen.- Se evalúa el desempeño de la primera reserva marina de Chile, creada en La Rinconada, Antofagasta, en base a los indicadores bióticos (abundancia; estructura poblacional y éxito del reclutamiento) de su especie foco de conservación, el pectínido A. purpuratus. Se utilizó información de la población de esta especie en 2001, 2002, 2005 y 2009, obtenida mediante evaluación directa por buceo, y del asentamiento de semilla sobre colectores artificiales. Los resultados muestran variación en la densidad de los ejemplares, que repercute fuertemente en la abundancia total de la población, con una disminución significativa de su talla media, y de los individuos sobre la talla mínima legal $(90 \mathrm{~mm})$ que se redujeron de 2,6 × $10^{6}$ en 2001 a $1,5 \times 10^{5}$ en 2009 . Esta reducción ha generado una disminución drástica de su stock desovante, afectando seriamente la captación de semilla, que ha descendido de un máximo de 15.340 ind. colector $^{-1}$ el 2001, a 1.157 ind. colector ${ }^{-1}$ el 2009 . El análisis de estos resultados bajo el prisma de 3 indicadores, señalan que la reserva no ha logrado recuperar su densidad ni su estructura de talla, para potenciar la disponibilidad de semilla y de adultos reproductores, planteado como su principal objetivo de creación. Si bien la razón principal de su creación fue la conservación del recurso A. purpuratus, esta medida se ha traducido en un aumento de interés por explotarlo ilegalmente. Se concluye la necesidad de implementar medidas que permitan una participación efectiva, y con equidad respecto a la utilización del recurso, considerando que luego de más de 20 años de veda, las medidas regulatorias impuestas por el Estado, no han tenido los resultados esperados.
\end{abstract}

Palabras clave: Desempeño, Reserva Marina, Argopecten purpuratus, Chile

\section{INTRODUCCIÓN}

En numerosos países donde los Pectínidos figuran entre los principales productos acuícolas, se han implementado rigurosas medidas de gestión de pesca, debido a la dramática disminución que han sufrido los bancos naturales, generando desventajas para su reclutamiento, y para el abastecimiento de semilla destinada al desarrollo de actividades de acuicultura intensiva o extensiva (Boucher \& Fifas 1995, Dickie 1995, MassoRojas 1996, Paulet et al. 1997). Dentro de estas medidas se 
puede nombrar el cierre a fines de 1994 de 3 áreas, en Georges Bank y al Sur de Nueva Inglaterra en USA, con una superficie de $17.000 \mathrm{~km}^{2}$, destinadas a proteger áreas de desove y producción de juveniles de peces bentónicos, y del sobreexplotado pectínido Placopecten magellanicus (Gmelin, 1791). Este cierre provocó en 1998 un aumento de 14 veces la biomasa de este pectínido, permitiendo nuevamente a partir de 1999 su extracción mediante rotación de áreas, manejo que como resultado mejoró su reclutamiento y conservación (Murawski et al. 2000). Otro ejemplo, se presenta en la zona costera de la Bahía de Gilbert, Canadá, donde el 2005 se creó la primera Área Marina Protegida (AMP), destinada a proteger la población residente de bacalao del Atlántico. En esta AMP a través de un co-manejo entre el organismo rector y la comunidad, se permite en zonas específicas la pesca comercial y de subsistencia del pectínido Chlamys islandica (Müller 1776). Sin embargo este tipo de pesca zonificada, ha generado una mortalidad por pesca diferencial, que ha provocado la eliminación de los individuos de grandes tallas (Wroblewski et al. 2009).

Chile también posee un largo historial de explotación por parte del sector pesquero artesanal de las poblaciones silvestres de Argopecten purpuratus (Lamarck, 1819). Sin embargo, bajos niveles de explotación caracterizaron esta pesquería hasta comienzos de los '80s, cambiando la situación drásticamente en 1984, donde se registró el máximo desembarque que alcanzó 4.997 ton (peso fresco), provenientes mayoritariamente de los bancos naturales ubicados en la región de Antofagasta como Mejillones y La Rinconada (Avendaño \& Cantillánez 1997). La presión de pesca continuó en los años siguientes, causando una disminución alarmante de los stocks naturales, que provocaron el cierre de su pesquería en 1988 (Avendaño \& Cantillánez 1997, Stotz \& Mendo 2001). A pesar de esta prohibición, el recurso continuó siendo extraído ilegalmente, produciéndose una delicada situación de degradación y colapso de sus bancos, que amenazó el desarrollo de la naciente actividad de cultivo, que dependía de la semilla obtenida por captación natural. La promulgación de la ley de pesca y acuicultura en 1991 pareció dar una oportunidad para la conservación de las especies comerciales, entre las cuales se encontraba A. purpuratus, incorporando la figura de Reserva Marina, como parte de las medidas de administración de los recursos hidrobiológicos chilenos. Estas reservas, que se crearían dentro del área reservada a la pesca artesanal, fueron declaradas como áreas de resguardo de los recursos hidrobiológicos, con el objeto de proteger, entre otros, las áreas de repoblamiento por manejo. Bajo este marco normativo, se promovió la conservación y restauración de los principales stocks silvestres y de su diversidad genética, con el fin de sustentar actividades comerciales claves para el país, entre ellos los cultivos. Ello, junto a antecedentes que daban cuenta que en el sector de La Rinconada, ubicada al Norte de la ciudad de Antofagasta, se sostenía el único banco natural de $A$. purpuratus, de alto potencial biológico, y capaz de ser recuperado (Avendaño \& Cantillánez 1997), permiten mediante Decreto Supremo No 522 de septiembre de 1997, la creación de la primera reserva marina en Chile en un área de 322 ha.

Si bien, las áreas marinas protegidas (AMPs) han sido consideradas como una de las más poderosas herramientas disponibles para combatir la sobreexplotación de los recursos marinos así como la degradación de hábitats oceánicos, también presentan ciertos riesgos o deficiencias en su designación como tal, que repercuten peligrosamente en su administración y en la opinión pública. Entre ellas, al generarse falsas expectativas de protección, como las que se indican para algunas de estas áreas, que ilusionan falsamente a los usuarios (Agardy et al. 2011). La reserva marina de La Rinconada, que a la fecha se encuentra bajo la tuición del Servicio Nacional de Pesca, cuenta con un sistema de vigilancia permanente, a través de guardias privados contratados por este Servicio. Dotada de este sistema de protección, esta reserva tiene como objetivo principal 'recuperar el banco natural, incluyendo abundancia total, densidad y estructura de tallas tal que pueda potenciarse la disponibilidad de semillas y de adultos reproductores, tanto al interior de la reserva como hacia fuera' $\left(\right.$ SUBPESCA 1997) ${ }^{1}$. En este escenario, y a más de una década de su creación, se evaluó el logro de dicho objetivo, y su desempeño como reserva marina, a través de 3 indicadores bióticos, tales como: i) abundancia de especies clave; ii) estructura poblacional de la especie clave, y iii) éxito del reclutamiento que se aplican para evaluar la efectividad de la gestión de AMPs (Pomeroy et al. 2006), utilizando para ello, información obtenida en la última década.

\section{Materiales Y MÉTODOS}

\section{Área de ESTUdio}

El área de estudio se localiza en el sector de La Rinconada (2328'28' 'S; 70³0’35' W), a $20 \mathrm{~km}$ al norte de la ciudad de Antofagasta, Chile (Fig. 1). En este sector la especieArgopecten purpuratus se distribuye entre los 6 y $29 \mathrm{~m}$ de profundidad (Cantillánez 2000). A macro escala, la zona corresponde a un ambiente de transición sub-tropical. Su orientación geográfica

${ }^{1}$ SUBPESCA. 1997. Determinación de reserva marina de ostión del norte (Argopecten purpuratus) en La Rinconada (II Región). Informe Técnico (R. Pesq.) 3: 1-23 (No publicado). Subsecretaría de Pesca, Gobierno de Chile, Valparaíso. 

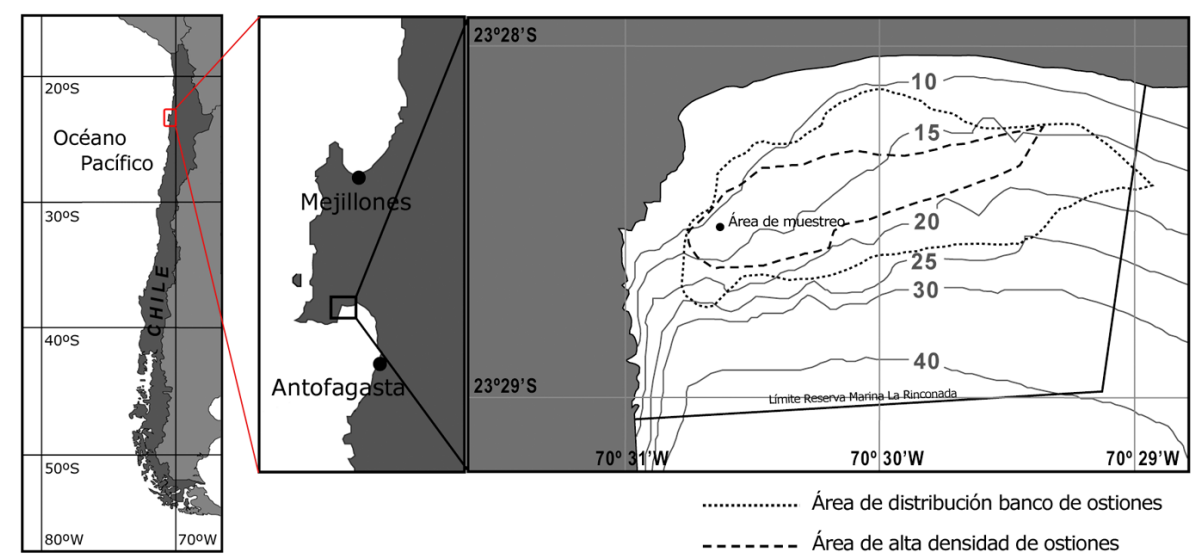

Figura 1. Localización geográfica de la reserva marina La Rinconada (2328'28'S, 70³0'35'W), $20 \mathrm{~km}$ al norte de la ciudad de Antofagasta-Chile. Se muestra la distribución de la población de A. purpuratus. Área de muestreo=sitio donde se registró la temperatura de fondo (16 m), y la captación de semillas de A. purpuratus / Geographic location of La Rinconada marine reserve $\left(23^{\circ} 28^{\prime} 28^{\prime \prime} \mathrm{S}, 70^{\circ} 30^{\prime} 35^{\prime \prime} \mathrm{W}\right), 20 \mathrm{~km}$ north of the City of Antofagasta, Chile. Distribution of the population of A. purpuratus. Sample area= site for bottom-water temperature recording (16 $\mathrm{m}$ depth), and spat collection of $A$. purpuratus

hacia el Sur y Suroeste y su posición latitudinal la exponen a la confluencia de varios tipos de masas de agua. Entre ellas, predomina en años normales, la masa de agua Sub-Antártica (ASA), que domina los $200 \mathrm{~m}$ superiores de la rama norte de la corriente fría de Humboldt. Las aguas ASA se mezclan con una menor proporción de aguas subtropicales (AST), de mayor salinidad y temperatura y también periódicamente con aguas más frías que provienen de mayor profundidad, correspondientes a aguas ecuatoriales sub-superficiales (AESS), las cuales ascienden hacia la costa debido a procesos de surgencia inducida por los vientos Sur y Suroeste (Escribano et al. 1995). Estos fenómenos que ocurren en el área, con la presencia de aguas AESS y ASA o de entremezcla, durante primavera y verano, y algunas veces en otoño, permiten la incorporación de suficientes nutrientes a los niveles superiores, permitiendo la existencia de una comunidad fitoplantónica típica de áreas de surgencia (Avaria \& Muñoz 1982, Rodríguez 1987, Rodríguez \& Escribano 1996). A micro escala, esta área se caracteriza por estar sujeta a flujos alternados de corriente, que resultan de una corriente predominante hacia el norte, pero que se encuentra con la barrera impuesta por la playa, reflejándose en forma cíclica en escalas de tiempo diarias. Ello produce un intercambio y mezcla de masas de aguas, y retención de material particulado en suspensión, incluyendo larvas de $A$. purpuratus, en un área de $5 \mathrm{~km}$ de radio (Cantillánez 2000, Avendaño et al. 2004, Cantillánez et al. 2007). En esta Bahía se ha observado además, una fuerte mezcla estacional de invierno que es causada por el viento, generando que la isoterma de los $13 \mathrm{o} 14^{\circ} \mathrm{C}$ ascienda muy cerca de la superficie (Escribano et al. 1995, Rodríguez \& Escribano 1996).

\section{Temperatura Del AGUA DE FONDO}

Se registró la temperatura del agua, a una profundidad de 16 $\mathrm{m}$, en el área de muestreo (Fig. 1) utilizando para ello un registrador de temperatura mini LOG 8-BIT(Vemco modelo TR). Los registros fueron tomados cada $6 \mathrm{~h}$, obteniéndose de ellos un promedio diario.

\section{INDICADORES DE DESEMPEÑO}

Como las AMP son una herramienta que permiten conservar las condiciones biofísicas de nuestros océanos y costas, usar indicadores para registrar estas condiciones, ha sido considerado de primordial interés para evaluar la efectividad de una AMP en cumplir sus objetivos. Es así como dentro de los 10 indicadores biofísicos definidos por Pomeroy et al. (2006), los primeros 6 se han utilizado para evaluar el contexto biótico dentro de estas áreas y su entorno, siendoi) abundancia de especies clave; ii) estructura poblacional de las especies clave; iii) distribución y complejidad del hábitat; iv) composición y estructura de la comunidad; v) éxito del reclutamiento dentro de la comunidad; y vi) integridad de la trama trófica. De estos, los 2 primeros permiten examinar el estado de las poblaciones. Los 4 restantes se usan para caracterizar las condiciones ecológicas y se ubican entre los indicadores más complejos de medir.

Tomando en consideración que la reserva marina La Rinconada, fue creada principalmente para conservar $A$. purpuratus como especie focal, cuyo manejo debiera ser de interés prioritario, se utilizaron 3 de los indicadores señalados, 
para examinar su estado poblacional (abundancia, estructura poblacional y éxito del reclutamiento), a objeto de hacer una estimación preliminar de la efectividad de esta reserva después de más de una década de su creación. El monitoreo de los cambios en la abundancia poblacional (el primero de estos indicadores), es considerado una actividad comúnmente realizada en la administración de un AMP, con el objeto de evaluar su protección, mejoramiento y/o mantenimiento; el segundo de ellos (conocer la estructura poblacional de la especie), sirve para indicar si la población tiene el potencial para subsistir en el tiempo, considerando que para ello, debe existir una cantidad apropiada de adultos reproductores; mientras que éxito de reclutamiento se entiende como el grado de siembra y asentamiento de larvas, de reclutamiento y supervivencia de juveniles, y sirve como sustituto de la capacidad de la población de persistir en el tiempo y ser viable (Pomeroy et al. 2006).

\section{Estimación de la ABUNDANCIA de $\boldsymbol{A}$. PURPURATUS}

Durante marzo 2001, mayo 2002, agosto 2005 y mayo 2009, se estimó la abundancia del recurso, a través de buceo autónomo (Avendaño \& Cantillánez 2005). Previamente, se estableció el perímetro de la superficie de distribución, delimitándola a través de la instalación de boyas indicadoras, georreferenciadas con ayuda de GPS. Su posterior proyección sobre el plano de la reserva, ayudado por un planímetro polar con compensación ZETING KP-27, permitió calcular la superficie en la que se distribuían los organismos (Avendaño \& Cantillánez 2005).

Delimitada la superficie de distribución, se realizó un muestreo piloto, para determinar el número mínimo de unidades de muestreo requeridas, estimar la densidad y abundancia total de la población de A. purpuratus presente (Thompson 1992). Para ello se aplicó la metodología señalada por Avendaño \& Cantillánez (2005), predefiniendo una grilla de muestreo simétrico, estableciéndose estaciones de muestreo que representaron el $10 \%$ de la superficie total que ocupó el banco cada año.

Resultados preliminares, permitieron definir 2 estratos en el área de distribución de $A$. purpuratus, diferenciados entre sí, por la heterogeneidad de varianzas de su densidad media. En consecuencia la evaluación final de la abundancia de la población, se realizó aplicando un muestreo al azar estratificado, estableciéndose muestreos aleatorios simples en cada estrato. Conocido el número mínimo de muestras a realizar, éstas se repartieron de acuerdo a la asignación diferencial en función del peso de cada estrato (superficie), muestreándose al azar
10 cuadrantes de $1 \mathrm{~m}^{2}$ cada una, dentro de áreas de $70 \times 70 \mathrm{~m}$ que representaron cada estación de muestreo (Avendaño \& Cantillánez 2005). Los ejemplares contabilizados fueron todos aquellos visibles y que se encontraban sueltos sobre el fondo. Posteriormente, en cada estrato se calculó el valor promedio de la densidad y la abundancia, de acuerdo a Thompson (1992). La estructura de talla de la población fue obtenida muestreando los organismos presentes en 5 réplicas de $1 \mathrm{~m}^{2}$, midiéndose su longitud máxima con una precisión de $0,1 \mathrm{~mm}$. Los datos obtenidos, fueron tabulados para conocer la frecuencia por talla, la cual se graficó con el fin de conocer como estaban representados los distintos grupos de talla dentro del área de distribución.

\section{Biomasa total Desovante}

La determinación de la fracción del stock desovante durante 2001, 2002, 2005 y 2009, medida en biomasa, se realizó multiplicando la biomasa total a la talla por la proporción de ejemplares maduros a la talla, siendo ésta última determinada por la función logística: $P\left(I_{50}\right)=1 \cdot\left(1+e^{\left(6,678-0,114^{*} \text { talla }\right)}\right)^{-1}$, calculada mediante un ajuste no lineal para la población de $A$. purpuratus de esta reserva marina (Avendaño et al. 2008²). Para la estimación en peso de la talla media (Lt) a cada intervalo de longitud (rango de $5 \mathrm{~mm}$ ), se utilizó la función potencial: $W t=0,0001 \cdot L t^{3,0416}(\mathrm{r}=0,9912)$, modelada para la misma población por Avendaño \& Cantillánez (2008). Posteriormente, a fin de comparar los valores interanuales de la biomasa desovante presente en el banco, se aplicó un análisis de varianza $(P<0,05)$, y se realizó un test a posteriori de Tukey. Los análisis se ejecutaron utilizando el software Minitab® 17.1.0.

\section{Captación de semillas}

La fijación de semilla sobre colectores, ha sido utilizada como un buen indicador del pre-reclutamiento natural, así como para estimar la variabilidad interanual de la abundancia de los prereclutas de Pectínidos como es el caso de Pecten maximus (Linnaeus, 1758) y Pecten alba (Tate, 1887) (Boucher 1985, Gwyther \& Burgess 1987, Sause et al. 1987, Thouzeau \& Lehay 1988, Thouzeau 1991a, b, c), por lo que se utilizó como indicador de reclutamiento en el presente trabajo. Para ello, desde una línea madre instalada de acuerdo a Thouzeau (1991a), dentro del área identificada como de mejor captación (Cantillánez 2000), entre abril y noviembre del 2001, 2002, 2005 y 2009 , se colocaron y reemplazaron por períodos de inmersión que duraron entre 21 y 57 días, 3 colectores japoneses para semilla de pectínidos 3D, quedando a $1 \mathrm{~m}$ del

${ }^{2}$ Avendaño M, M Ortiz, M Cantillánez \& J Soza. 2008. Determinación de escenarios para la explotación sustentable de la Reserva Marina La Rinconada. Informe Final, Proyecto Innova/CORFO cód. 04CR71PM-01. Universidad de Antofagasta, Antofagasta, 121 pp. 
fondo, en una columna de agua de $17 \mathrm{~m}$. Estos colectores se constituyeron por una bolsa plástica externa de 0.5 x $1 \mathrm{~mm}$ de tamaño de malla, relleno con una red de Netlón $®$ de $10 \mathrm{~mm}$ de tamaño de malla. Su extracción se realizó mediante buceo, envolviéndolos individualmente en bolsas protectoras y transportándolos hasta el laboratorio, donde se lavaron cuidadosamente, recolectándose toda la semilla presente en cada uno de ellos. Un tamiz de $180 \mu \mathrm{m}$ fue utilizado en el lavado para evitar pérdidas de post-larvas. Se contaron y midieron todas las semillas fijadas por colector cuando sus abundancias fueron bajas, sin embargo, cuando fueron abundantes, éstas se homogenizaron dentro de un muestreador de plancton de 10 divisiones, y 2 submuestras fueron tomadas para contarlas y medirlas en un estereomicroscopio con ocular graduado. El número de semilla fijada se obtuvo promediando la cantidad encontrada en los 3 colectores (Avendaño et al. 2006). Posteriormente se aplicó el programa MIX 3.1.a (Mac Donald \& Pitcher 1979), a la distribución de frecuencia de los datos de talla de las semillas fijadas en el período de inmersión, para separar micro-cohortes. Los histogramas de frecuencia de talla construidos fueron analizados siguiendo una distribución normal. El análisis entrega el promedio de talla, la varianza y las proporciones de cada micro-cohorte identificada, junto con estimar el valor de $P$. El nivel de significancia utilizado fue de 0,05 .

\section{Resultados}

\section{Temperatura DEL AGUA DE FONDO}

A pesar que no fue posible registrar durante los años de estudio (2001, 2002, 2005 y 2009) datos de temperatura entre abril- agosto de 2005 y abril-julio de 2009, los ciclos térmicos registrados (Fig. 2) dieron cuenta de un patrón anual estacional marcado por el alza de temperaturas medias durante el periodo estival (diciembre-marzo), la que regularmente se elevó hasta $18^{\circ} \mathrm{C}$. Una estrecha variación de éstas en el área de la reserva, se mantuvo durante otoño e invierno, fluctuando entre 14 y $15^{\circ} \mathrm{C}$, tendencia que regularmente se rompió al iniciarse primavera (septiembre), donde se aprecian al menos una abrupta baja térmica, hasta $13^{\circ} \mathrm{C}$ que regularmente marcó alzas posteriores de temperatura producidas en cortos lapsos de tiempo. Los registros de temperatura a intervalos de $6 \mathrm{~h}$, mostraron variaciones intradiarias de hasta $5^{\circ} \mathrm{C}$. Esta gran dispersión de la temperatura fue marcada en primavera-verano y fue menos intensa en otoño-invierno.

\section{DistribuCión Y ABUNDANCIA DEL BANCO}

Las evaluaciones realizadas entre 2001 y 2009, indican que el área de distribución del banco de A. purpuratus de La Rinconada, varió entre 278,4 y 237,5 ha, extendiéndose entre

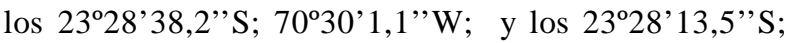
$70^{\circ} 29^{\prime} 7,9^{\prime \prime} \mathrm{W}$, en profundidades de 6 a $29 \mathrm{~m}$. Dentro de estas áreas, los ejemplares se distribuyeron de forma estratificada, concentrándose en una superficie que varió según los años, entre 71,3 y 51,1 ha, en veriles de 7 a $17 \mathrm{~m}$ de profundidad. En esta superficie se registraron además, variaciones en la densidad media del recurso, reduciéndose de 12,08 ind. $\mathrm{m}^{-2}$ que presentaba en el 2001 a 7,43 ind. $\mathrm{m}^{-2}$ en el 2005. Un incremento a 11,6 ind. $\mathrm{m}^{-2}$ ocurrió en 2009, que repercutió en un aumento de la abundancia total de la población en ese año (Tabla 1).

Tabla 1. Densidad y abundancia de A. purpuratus en el banco de la reserva marina La Rinconada, Antofagasta, Chile, en marzo 2001; mayo 2002; agosto 2005 y mayo 2009. H =área de alta densidad (zona central), L=área de baja densidad (periférica). DE=desviación estándar, SV= varianza media estratificada / Density and abundance of A. purpuratus in the marine reserve La Rinconada, Antofagasta, Chile, in March 2001; May 2002; August 2005 and May 2009. $\mathrm{H}=$ high density area (central zone), L= low density area (peripheral). SD= standard deviation, $\mathrm{SV}=$ mean stratified variance

\begin{tabular}{|c|c|c|c|c|c|c|c|c|c|c|}
\hline \multirow{2}{*}{ Año } & \multirow{2}{*}{$\begin{array}{c}\text { No. } \\
\text { Cuadratas }\end{array}$} & \multicolumn{3}{|c|}{ Estrato $\mathrm{H}$} & \multicolumn{3}{|c|}{ Estrato L } & \multicolumn{3}{|c|}{ Total } \\
\hline & & $\Sigma$ ostiones & ind. $m^{-2}$ & $\mathrm{DE}$ & $\Sigma$ ostiones & ind. $m^{-2}$ & $\mathrm{DE}$ & $\sum$ ostiones & $\mathrm{DE}$ & SV \\
\hline 2001 & 993 & 6.086 .383 & 12,08 & 8,55 & 4.044 .374 & 1,98 & 2,91 & 10.130 .758 & 367.057 & 0,020 \\
\hline 2002 & 871 & 6.433 .758 & 9,01 & 8,19 & 1.814 .508 & 1,05 & 1,61 & 8.239 .242 & 333.950 & 0,019 \\
\hline 2005 & 934 & 5.542 .780 & 7,43 & 7,37 & 1.791 .900 & 1,10 & 1,45 & 7.334 .680 & 415.129 & 0,025 \\
\hline 2009 & 1.380 & 5.901 .075 & 11,60 & 9,94 & 3.053 .895 & 1,34 & 1,89 & 8.954 .969 & 333.753 & 0,014 \\
\hline
\end{tabular}




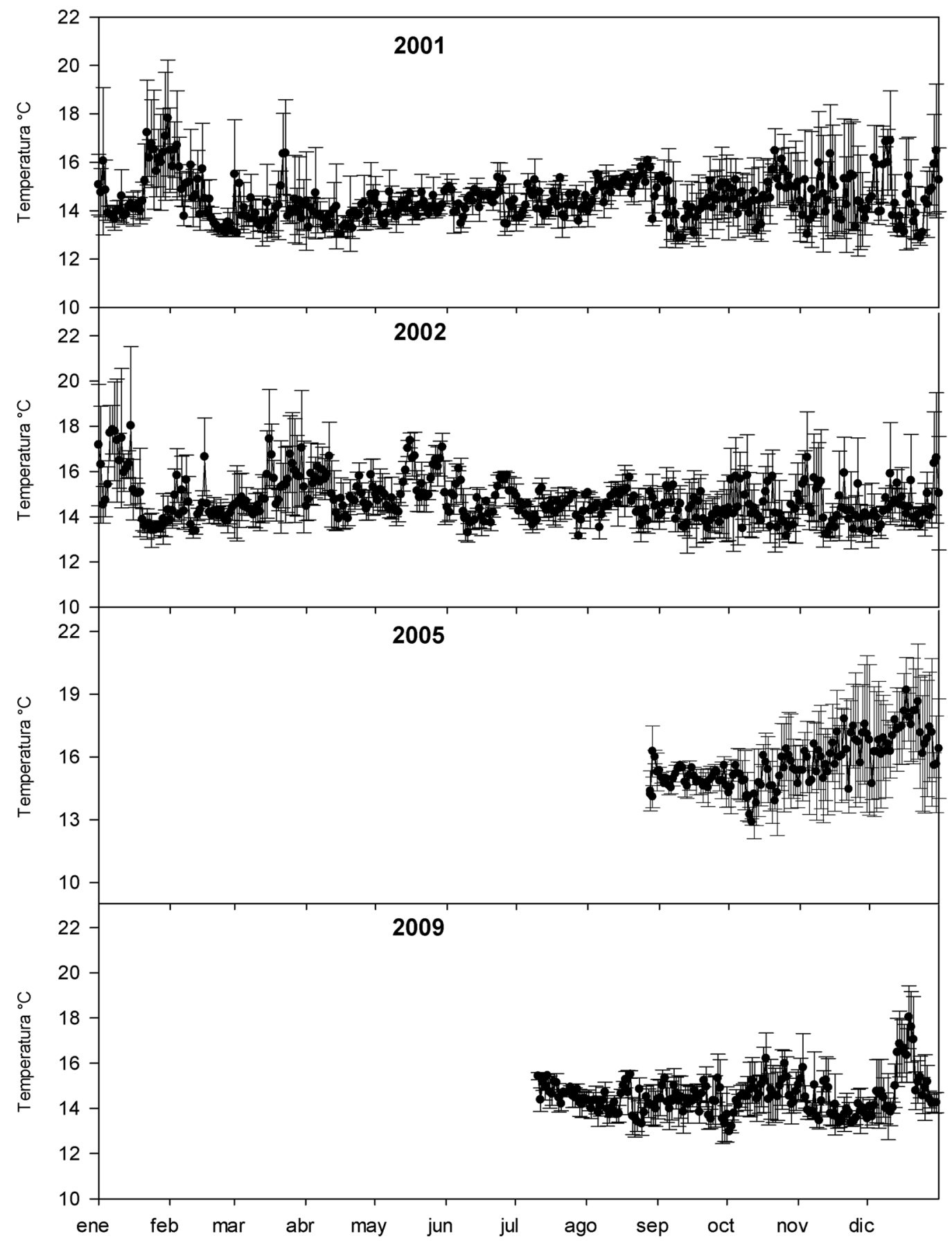

Figura 2. Promedio diario de temperatura del agua $\left({ }^{\circ} \mathrm{C}\right)$, obtenidas con un registrador de temperatura Minilog 8-BITVemco, a $16 \mathrm{~m}$ de profundidad en el área de muestreo de la reserva marina La Rinconada (ver Fig. 1 para ubicación del sitio). Barras grises muestran la desviación estándar / Average daily water temperature $\left({ }^{\circ} \mathrm{C}\right.$ ) obtained with a Minilog 8-BIT Vemco temperature recorder, at $16 \mathrm{~m}$ depth in the sampling area at La Rinconada marine reserve (see Fig. 1 for site location). Gray bars= standard deviation 


\section{Estructura de TALLA DEL BANCo}

El análisis global de la estructura demográfica que presentó la población (Fig. 3), mostró una disminución significativa de la talla media de los individuos, que se tradujo en una disminución importante de los individuos sobre la talla mínima legal $(90 \mathrm{~mm})$ que formaron parte del total de la población en esos años (Tabla
2), reduciéndose del 25,8 al 11,3\% entre el 2001 y 2002, y al $0,85 \%$ para el 2005. Un pequeño incremento de estos ejemplares, que alcanzó al 1,7\% del total de individuos, se produjo entre el 2005 y 2009 (Tabla 2).

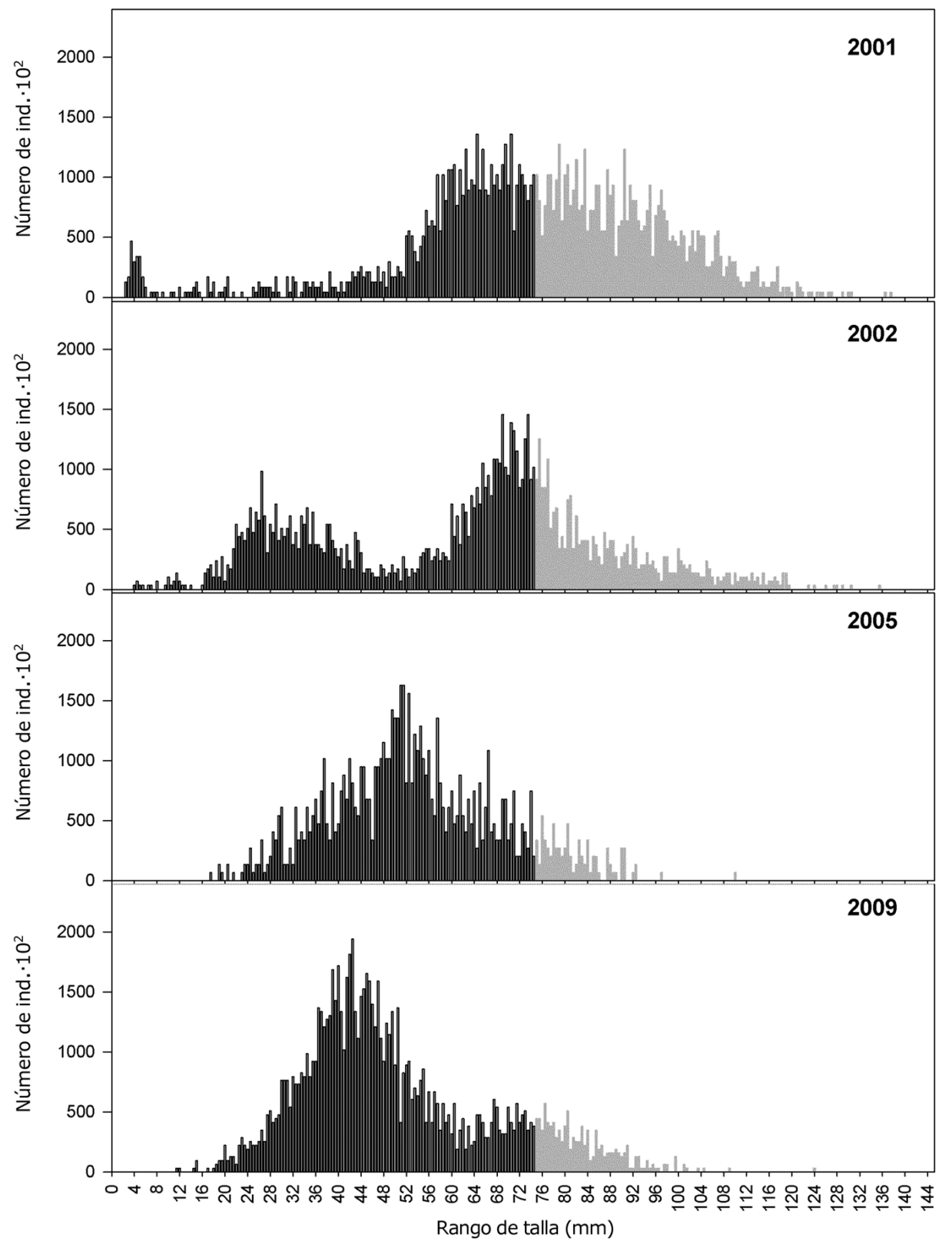

Figura 3. Distribución de frecuencia de talla (número de ind. · $10^{2}$; longitud en $\mathrm{mm}$ ) de A. purpuratus, en la población de la reserva marina La Rinconada en marzo 2001, mayo 2002, agosto 2005 y mayo 2009. Barras negras: muestran los individuos de 1 año de edad / Size frequency distribution (numbers of ind. $\cdot 10^{2}$; shell length in $\mathrm{mm}$ ) of the population of A. purpuratus at La Rinconada marine reserve in March 2001, May 2002, August 2005 and May 2009. Black bars= oneyear-old individuals 
Tabla 2. Rango de talla (ancho) y talla media ( $\pm D E$ ) de A. purpuratus en la población de la reserva marina La Rinconada, durante los 4 años de observaciones. Números de ejemplares de talla mínima legal (TML $\geq$ $90 \mathrm{~mm}$ ). Fertilidad media (número de ovocitos liberados en función de la talla media, ver Avendaño et al. (2001) / Size range (width) and mean shell size $( \pm S D)$ in the A. purpuratus population at La Rinconada Marine Reserve during 4 years of observations. Numbers of legalsized scallops (TML $\geq 90 \mathrm{~mm}$ ). Mean Fertility (number of oocytes released by average-sized individuals, see Avendaño et al. (2001)

\begin{tabular}{lrrrrr}
\hline Año & $\begin{array}{c}\text { Rango talla } \\
(\mathrm{mm})\end{array}$ & $\begin{array}{c}\text { Media } \\
(\mathrm{mm})\end{array}$ & DE & $\begin{array}{c}\text { Individuos } \\
\text { TML }\end{array}$ & $\begin{array}{c}\text { Fertilidad } \\
\text { Media * }\end{array}$ \\
\hline 2001 & $10,2-137,2$ & 75,79 & 9,82 & $2,6 \cdot 10^{6}$ & $13,5 \cdot 10^{6}$ \\
2002 & $10,0-135,2$ & 62,08 & 4,47 & $9,3 \cdot 10^{5}$ & $6,2 \cdot 10^{6}$ \\
2005 & $4,5-109,7$ & 50,16 & 17,88 & $6,2 \cdot 10^{4}$ & $2,2 \cdot 10^{5}$ \\
2009 & $11,0-123.9$ & 49,90 & 16,77 & $1,5 \cdot 10^{5}$ & $2,2 \cdot 10^{5}$ \\
\hline
\end{tabular}

\section{Biomasa total Desovante}

En el 2001, la biomasa total desovante en el banco alcanzó 563,14 ton, donde los individuos con una talla de $92,4 \mathrm{~mm}$ hicieron el mayor aporte de gametos. En los años posteriores se registró una fuerte disminución del stock desovante, junto a una drástica reducción de la talla de los individuos que realizan el mayor aporte de gametos al banco, que alcanzó los 72,4 mm en 2002 y 2005, respectivamente (Fig. 4). En el 2009, se produjo un leve incremento en la talla de estos individuos, alcanzando 77,3 mm, sin embargo, la biomasa del stock desovante disminuyó a 114,93 ton, representando solo el 20,4\% de lo estimado para el 2001 (Fig. 4). Los resultados del ANDEVA, mostraron que la biomasa total desovante presenta diferencias significativas entre los periodos anuales de estudio $(P=0,0001)$, diferencias que son explicadas por el año 2001 $(P<0,05)$

Figura 4. Biomasa desovante (ton) de ejemplares maduros a la talla, presente en el banco de ostiones de La Rinconada, en marzo 2001, mayo 2002, agosto 2005 y mayo 2009 / Spawning Biomass (ton) of mature scallops at the size, estimated for the population of La Rinconada marine reserve in March 2001, May 2002, August 2005 and May 2009

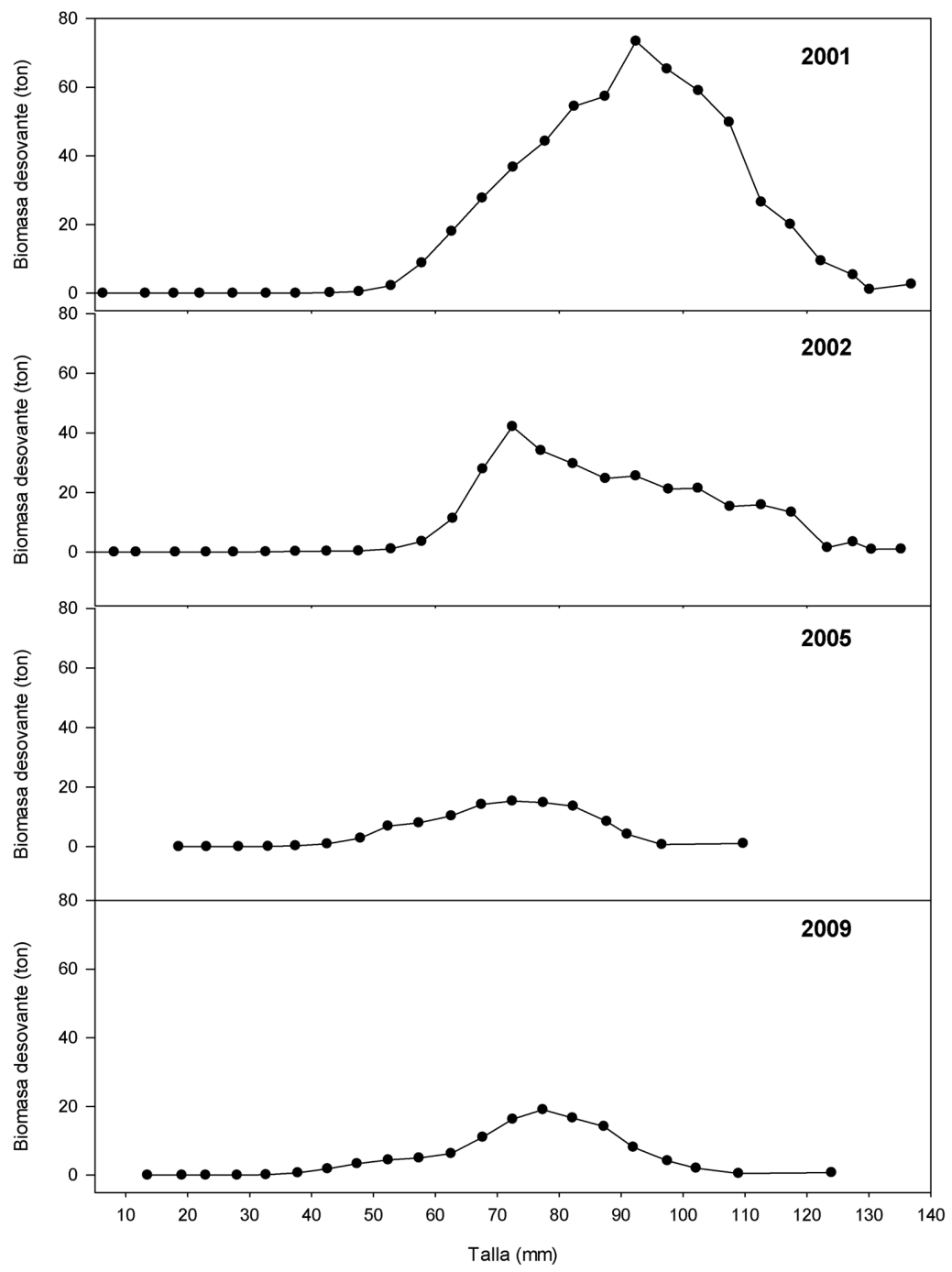




\section{Captación de semillas}

Los resultados de captación de semilla en los 4 años de estudio, mostraron que el asentamiento de post-larvas en las unidades de captación, ocurrió durante todo el periodo de prueba, y que las mayores fijaciones se produjeron entre septiembre y noviembre (Tabla 3). Sin embargo, los resultados también muestran una fuerte reducción en el número de semillas fijadas entre 2005 y 2009, respecto de las obtenidas el 2001 y 2002. En estos 2 últimos años, los valores máximos alcanzados fueron de 15.340 y 11.730 ind. colector ${ }^{-1}$ respectivamente, mientras que el 2005 y 2009 se lograron 800 y 1.157 ind. colector $^{-1}$ respectivamente. La aplicación del programa MIX sobre la estructura de talla de estas semillas, discriminó entre 1 y 3 micro cohortes de post larvas asentadas sobre los colectores, durante los diferentes períodos de inmersión, presentando tallas medias que variaron entre 434 y $5.377 \mathrm{~mm}$ (Tabla 3).

\section{Discusión}

Los registros de temperatura mostraron una variabilidad permanente en este ecosistema, con fuertes variaciones intradiarias que alcanzaron hasta $5^{\circ} \mathrm{C}$ en los períodos estudiados. Estas variaciones intradiarias, han sido correlacionadas con el alto grado de actividad reproductiva que presenta la población de ostiones que allí se distribuye (Cantillánez et al. 2005), dando origen a continuos pool larvales que culminan en su fijación, como se corrobora a través de los colectores en el presente estudio.

Los resultados mostraron que la población de A. purpuratus de la reserva se distribuye en forma agregada, lo que ya se ha señalado (Avendaño \& Cantillánez 1996), y común a lo que presentan poblaciones de otras especies de pectínidos (Thouzeau \& Lehay 1988, Caddy 1989). En la reserva esta
Tabla 3. Fijación de semilla de $A$ purpuratus sobre colectores artificiales en la reserva marina de La Rinconada, durante los períodos comprendidos entre abril y noviembre de 2001, 2002,2005 y 2009. Se muestra los períodos de inmersión de los colectores, el número promedio de semilla fijada, las micro cohortes discriminadas, su talla media y su desviación estándar (DE) / Spat settlement of A. purpuratus on artificial collectors at La Rinconada marine reserve, during the periods April -November 2001, 2002, 2005 and 2009. Collectors' immersion periods are shown, as well as average number of spat fixed, the discriminated pseudo cohort, the mean length and standard deviation (SD)

\begin{tabular}{|c|c|c|c|c|c|}
\hline $\begin{array}{l}\text { Fecha instalación } \\
\text { y extracción }\end{array}$ & $\begin{array}{l}\text { Días de } \\
\text { inmersión }\end{array}$ & $\begin{array}{l}\text { Promedio de semilla } \\
\text { por colector } \pm \mathrm{DE}\end{array}$ & $\begin{array}{c}\text { Cohorte } 1 \\
\text { talla } \pm \mathrm{DE}(\mu \mathrm{m})\end{array}$ & $\begin{array}{c}\text { Cohorte } 2 \\
\text { talla } \pm \text { DE } \\
(\mu \mathrm{m})\end{array}$ & $\begin{array}{c}\text { Cohorte } 3 \\
\text { talla } \pm \mathrm{DE} \\
(\mu \mathrm{m})\end{array}$ \\
\hline $06 / 04 / 01-06 / 05 / 01$ & 30 & $1.170 \pm 197$ & $702 \pm 160$ & & \\
\hline $06 / 05 / 01-08 / 06 / 01$ & 33 & $878 \pm 137$ & $1.618 \pm 272$ & $841 \pm 223$ & \\
\hline 08/06/01 - 06/07/01 & 28 & $1.450 \pm 473$ & $1.125 \pm 158$ & $532 \pm 112$ & \\
\hline 06/07/01 - 09/08/01 & 34 & $72 \pm 31$ & $502 \pm 116$ & & \\
\hline 09/08/01 - 14/09/01 & 36 & $798 \pm 101$ & $458 \pm 166$ & & \\
\hline $14 / 09 / 01-05 / 10 / 01$ & 21 & $200 \pm 28$ & $1.067 \pm 133$ & & \\
\hline $05 / 10 / 01-15 / 11 / 01$ & 41 & $15.340 \pm 2.786$ & $3.200 \pm 290$ & $1.740 \pm 280$ & $700 \pm 170$ \\
\hline $07 / 04 / 02-14 / 05 / 02$ & 37 & $400 \pm 35$ & $1.680 \pm 360$ & & \\
\hline $14 / 05 / 02-14 / 06 / 02$ & 31 & $585 \pm 77$ & $1.991 \pm 307$ & & \\
\hline $14 / 06 / 02-05 / 07 / 02$ & 21 & $400 \pm 138$ & $1.687 \pm 554$ & & \\
\hline 05/07/02 - 23/08/02 & 49 & $600 \pm 39$ & $1.762 \pm 434$ & & \\
\hline $23 / 08 / 02-29 / 09 / 02$ & 37 & $1.410 \pm 212$ & $883 \pm 267$ & & \\
\hline $29 / 09 / 02-25 / 10 / 02$ & 26 & $11.730 \pm 1.151$ & $1.022 \pm 381$ & $434 \pm 56$ & \\
\hline $25 / 10 / 02-22 / 11 / 02$ & 28 & $3.009 \pm 495$ & $399 \pm 64$ & & \\
\hline $15 / 04 / 05-17 / 05 / 05$ & 32 & $95 \pm 31$ & $1.120 \pm 260$ & & \\
\hline $17 / 05 / 05-18 / 06 / 05$ & 32 & $11 \pm 2$ & $1.062 \pm 195$ & $426 \pm 98$ & \\
\hline $18 / 06 / 05-21 / 07 / 05$ & 33 & $4 \pm 0.6$ & $1.088 \pm 384$ & & \\
\hline $21 / 06 / 05-09 / 08 / 05$ & 49 & $145 \pm 22$ & $5.377 \pm 948$ & $2.272 \pm 508$ & \\
\hline 09/08/05 - 25/09/05 & 47 & $247 \pm 93$ & $2.967 \pm 590$ & $1.013 \pm 212$ & \\
\hline $25 / 09 / 05-24 / 10 / 05$ & 29 & $555 \pm 107$ & $905 \pm 233$ & $463 \pm 63$ & \\
\hline $24 / 10 / 05-28 / 11 / 05$ & 34 & $880 \pm 87$ & $1.793 \pm 428$ & $1.092 \pm 265$ & \\
\hline $24 / 04 / 09-29 / 05 / 09$ & 35 & $268 \pm 113$ & $2.500 \pm 490$ & $1.590 \pm 260$ & $490 \pm 100$ \\
\hline $29 / 05 / 09-10 / 07 / 09$ & 42 & $29 \pm 16$ & $590 \pm 110$ & & \\
\hline 10/07/09 - 05/09/09 & 57 & $12 \pm 2$ & $1.088 \pm 384$ & & \\
\hline 05/09/09 - 30/10/09 & 55 & $1.157 \pm 265$ & $1.290 \pm 210$ & & \\
\hline $30 / 10 / 09-28 / 11 / 09$ & 29 & $1.614 \pm 224$ & $1.087 \pm 226$ & $539 \pm 78$ & \\
\hline
\end{tabular}


zona de agregación, coincidió con la zona de distribución del alga rodofícea Rhodymenia sp., que se distribuyó entre 9 y 18 m de profundidad (Avendaño \& Cantillánez 1996, Cantillánez 2000, Cantillánez et al. 2001). Sin embargo, los resultados mostraron que esta área de agregación, se redujo a lo largo del estudio, y en ella, la densidad de ejemplares, afectando seriamente la abundancia del recurso. También se registró una disminución sostenida de su talla media, que entre el 2001 y 2009 se redujo de 76 a $50 \mathrm{~mm}$. Los ejemplares sobre la talla mínima legal (90mm), también mostraron una fuerte disminución que alcanzó al 97\% entre el 2001 y 2005, recuperándose en apenas un $6 \%$ el 2009. Estas variaciones registradas tanto en la estructura de talla, como en su abundancia, no obedecerían a mecanismos naturales de oscilación en el volumen de esta población, frecuentemente observada en otras poblaciones de bivalvos, y particularmente con los pectínidos (Orenzans et al. 1991a), sino probablemente a causas de origen antrópico como la pesca clandestina (Avendaño \& Cantillánez 2005), denunciada constantemente por los guardias de esta reserva. No obstante, es necesario señalar la necesidad de evaluar el impacto de la mortalidad natural de $A$. purpuratus en esta reserva, y su influencia en la variabilidad interanual de su abundancia. Actualmente la mortalidad natural no puede ser diferenciada de la mortalidad por pesca, ya que los infractores que realizan la extracción clandestina, desconchan y tiran las valvas vacías sobre el banco (Avendaño \& Cantillánez 1996, Avendaño et al. 2004 ${ }^{3}$ ). Los trabajos de Ortiz et al. (2009, 2010), apoyados en modelos teóricos multiespecíficos cuantitativos y cualitativos, con el objeto de establecer estrategias de manejo para los recursos comerciales de esta reserva marina, señalaron a través de la mortalidad por pesca al máximo rendimiento sostenible, que no es posible incrementar los niveles de extracción de A. purpuratus, debido que éste se encontraría sobreexplotado. De acuerdo a estos autores, el nivel actual de explotación está representado sólo por pesca ilegal, lo que limita el diseño de un plan de explotación sostenible al no dejar margen para fijar niveles de extracción legal de este recurso. La reducción de la talla media de A. purpuratus registrada en este estudio, a causa de la disminución de los ejemplares de tallas mayores, también ha sido reportada para Ch. islandica en zonas localizadas de un AMP de la Bahía de Gilbert (Canadá), donde la pesca autorizada provoca una mortalidad diferencial al extraerse los ejemplares más grandes (Wroblewski et al. 2009).

Al aplicar los parámetros de crecimiento obtenidos para esta población de A. purpuratus (Avendaño \& Cantillánez 2005), sobre la estructura de talla que ésta presentó en cada evaluación realizada, fue posible hacer una estimación de las pérdidas de ejemplares que ocurren en esta reserva. Estos parámetros permitieron señalar que los ejemplares menores a $80 \mathrm{~mm}$ encontrados en el 2002, correspondían a nuevos reclutas, no presentes en el banco en marzo de 2001 (6,5 x $10^{6}$ individuos). Descontando esta cifra, de los $8,2 \times 10^{6}$ ejemplares registrados el 2002, se obtuvo que sólo el $17,1 \%$, formaban parte de los $10,1 \times 10^{6}$ evaluados el 2001, yen consecuencia, entre ambos años se habrían perdido cerca de 8,4 × $10^{6}$ individuos de tallas mayores de la reserva. El mismo análisis, realizado sobre las estructuras de talla que presentó la población el 2005 y 2009 , señaló que la nueva clase anual alcanzó el 88,5\% de la población en el 2005 y 90,2\% en el 2009, y en consecuencia los individuos mayores a un año de edad, tuvieron una baja incidencia en la estructura poblacional de ambos años. La desaparición sistemática de los ejemplares más viejos y más grandes dan cuenta de la pesca ilegal que afecta a esta población, señalada por Ortiz et al. (2009, 2010).

Las fijaciones de semilla sobre colectores utilizadas como indicador del pre-reclutamiento, mostraron una fuerte reducción en los niveles de captación a medida que pasó el tiempo, disminuyendo en los últimos años en cerca del $90 \%$ de los períodos de mayor fijación registrados (2001-2002). Ello a pesar que A. purpuratus en esta reserva, presenta un proceso reproductivo continuo, y que el área posee la capacidad para retener sus larvas (Cantillánez et al. 2005, Avendaño et al. 2008a), obteniéndose en este estudio hasta 3 cohortes fijadas sobre colectores inmersos por períodos de 35 y 41 días. La disminución en los niveles de fijación, estaría relacionada con la reducción de los ejemplares por sobre la talla mínima legal, que son los que hacen el mayor aporte reproductivo (Avendaño et al. 2001), mostrando una relación denso dependiente entre abundancia de reproductores mayores a $90 \mathrm{~mm}$ con el número de semillas colectadas. La fuerte reducción de la biomasa total desovante que experimentó este banco en el período de estudio, junto a la reducción de la talla de individuos que realizan el mayor aporte de gametos estaría corroborando esta relación (Figs. 3 y 4). En los pectínidos, numerosos estudios indican la correlación existente entre el tamaño de la población desovante con el número de semillas colectadas (Orenzanz et al. 1991a, Bojórquez-Verástica 1997, Maeda-Martínez et al. 2001, Narvarte et al. 2001). Orensanz et al. (1991b) señalaron además, que cualquier relación entre tamaño poblacional y reclutamiento, incluyendo la fijación en colectores, es el resultado de diversos procesos que se coordinan en sus escalas espaciales y temporales. Mason (1983) por otro lado, indicó que los patrones de asentamiento de las semillas de pectínidos

\footnotetext{
${ }^{3}$ Avendaño M, M Cantillánez, L Rodríguez, O Zuñiga, R Escribano \& M Oliva. 2004. Conservación y protección Reserva Marina La Rinconada Antofagasta-Chile, 215 pp. Informe Final Proyecto FNDR Cód. BIP N²0127869-0, Universidad de Antofagasta, Antofagasta.
} 
dependerían de la distribución de sitios adecuados, mientras que Sinclair et al. (1985), señalan que la existencia permanente de bancos de estos organismos en un mismo lugar, es producto de la capacidad de estos de autocolonizarse, debido a su ubicación en áreas oceanográficas con la cualidad de retener sus larvas en el sitio, como se ha señalado para esta reserva marina.

El análisis de estos resultados bajo el prisma de los 3 indicadores seleccionados para evaluar la efectividad de esta reserva, indica que no se ha logrado recuperar la densidad ni la estructura de talla de la especie focal. Por el contrario, ambas se estarían deteriorando, impidiendo potenciar la disponibilidad de semillas y de adultos reproductores como proponía el principal objetivo de creación de la reserva. La población de A. purpuratus en esta área, por causas de intervenciones antrópicas, que no obedecen a ningún plan racional de manejo, presenta una talla media cada vez más pequeña, y un bajo número de ejemplares de talla mínima legal, que la exponen a una menor probabilidad de albergar el número necesario de reproductores para repoblarse y persistir en el tiempo (Pomeroy et al. 2006). Si bien la nueva clase anual, producto de: i) el ciclo reproductivo de la especie, ii) la talla en que el $50 \%$ de los individuos se encuentran sexualmente maduros $\left(I_{50 \%}=58,6\right.$ $\mathrm{mm}$ ), y iii) la capacidad del área de retener las larvas, que señalan Avendaño \& Cantillánez (2008), compensa parte de las pérdidas provocadas durante el año, la extracción sostenida y sistemática de los ejemplares de tallas mayores, no solo estarían incidiendo en la fertilidad de los individuos, sino que también en su selección genética. La extracción sistemática de individuos de mayor tamaño, dejando sobre el fondo los ejemplares de crecimiento lento, constituye en el mediano plazo, un mecanismo inverso de selección genética, como se ha observado en el cultivo de esta especie (Meffe \& Carrol 1994, Stotz 2000, Avendaño \& Cantillánez 2008).

A pesar que la razón de la creación de esta reserva marina, fue principalmente la conservación del recurso A. purpuratus, esta medida se ha traducido en un aumento del interés por parte de pescadores artesanales por explotarlo ilegalmente, reduciendo significativamente su abundancia y modificando su estructura de talla. El potencial incremento de la extracción ilegal de ostiones, sin un plan de manejo holístico de explotación, o de manejo inteligente está trayendo consecuencias negativas en la salud y futura trayectoria de este ecosistema. Esto a su vez podría provocar riesgos adicionales en el desarrollo de las actividades de cultivo de $A$. purpuratus en el país, ya que no existe otro banco con las características del que se localiza en esta reserva marina, que pudiera convertirse en un potencial abastecedor de semillas (Avendaño 1993, Avendaño \& Le Pennec 1996, 1997; Cantillánez 2000, Cantillánez et al. 2001, 2005, 2007; Avendaño \& Cantillánez 2005, Avendaño et al. 2006, 2008a, b; Thouzeau et al. 2001, Ortiz et al. 2009, 2010).

Sin embargo, también es necesario implementar medidas que permitan una participación efectiva, y con equidad para los pescadores artesanales respecto a la utilización del recurso, considerando que a casi 30 años de mantenerse en veda, estas medidas regulatorias impuestas por el Estado, no han tenido los resultados esperados. Se puede señalar a este respecto, de acuerdo a Morales \& Gezan (1986) que la aplicación de vedas y prohibiciones de comercialización de recursos pesqueros, llevan a ciertos pescadores a no respetarlas, producto de que un interés económico de corto plazo impuesto por la demanda primaría en ellos, por sobre una conciencia de protección racional de más largo plazo. Ello los impulsaría a transgredir, las actuales políticas de desarrollo de la pesca artesanal, que plantean como objetivo del ordenamiento de las pesquerías artesanales, y la conservación de los recursos marinos pesqueros en el largo plazo (SUBPESCA 1995) ${ }^{4}$. Blount \& Pitchon (en Jones 2009), a través de un análisis antropológico, señalaron que los pescadores están comprometidos con la pesca como una forma de vida, más que sobre la base del racionalismo económico, por loque a menudo ven los problemas de equidad, en términos de los efectos de las propuestas y políticas sobre su forma de vida, más que sólo en términos de la distribución de costos y beneficios.

Aunque el Plan General de Administración diseñado para esta reserva, contempla procesos de participación de los usuarios, así como la ejecución de actividades de educación y difusión de sus objetivos, y de divulgación de los planes de manejo, como actividades esenciales para disminuir las extracciones ilegales de los recursos de la que ha sido objeto, faltan medidas complementarias y/o sustitutas para los pescadores locales, así como en su educación, en el sentido propio del término. Cabe señalar, que dentro de las nuevas estrategias propuestas para un desarrollo sustentable, se indica el manejo adaptativo de ecosistemas sometidos a explotación, en el que se han incorporado a todos los usuarios, lo que permite corregir fallas en las acciones de manejo que puede presentar una especie, grupos de especies o el ecosistema, ante las incertezas que se generan como respuestas frente a las intervenciones humanas (Marín \& Delgado 1997). Por ello también, mediante el establecimiento de regímenes de co-

${ }^{4}$ SUBPESCA. 1995. Regulación del Recurso Ostión del Norte. Subsecretaría de Pesca-Ministerio de Economía Fomento y Reconstrucción. Informe Técnico 70: 1-31. (No publicado). Valparaíso. 
manejo, se han incorporado a los usuarios en el manejo de áreas marinas protegidas, garantizándoles facultades para participar en la planificación y manejo de estos ecosistemas, lo que ha resultado en un factor clave para su desarrollo (NurseyBray \& Rist 2009). Ello porque el co-manejo, permite un proceso continuo de solución de problemas, mediante una amplia deliberación y negociación en el marco de un acuerdo de poder compartido, en que se asumen responsabilidades, derechos y deberes, con un enfoque descentralizado para la toma de decisiones al involucrar a los usuarios locales en este proceso como iguales con el Estado (Carlssona \& Berkesb 2005). Los pescadores por su nivel cultural y umbral de pobreza, son muy pocos sensibles al discurso sobre la conservación del recurso, la biodiversidad, o el medio ambiente, sus preocupaciones son de otro orden, y es ahí precisamente, donde el ente administrador, debiera implementar políticas de participación efectivas como el co-manejo de esta reserva, para lograr sus objetivos, permitiendo de paso que la primera reserva marina de Chile también los cumpla.

\section{Agradecimientos}

El presente estudio fue desarrollado en el marco de los proyectos FNDR Código BIP N 20127869-0; CORFO N ${ }^{\circ}$ 04CR71PM-01 y FIP 2008-54.

\section{LITERATURA CITADA}

Agardy T, G de Sciara \& P Christie. 2011. Mind the gap: Addressing the shortcomings of marine protected areas through large scale marine spatial planning. Marine Policy 35: 226-232.

Avaria S \& P Muñoz. 1982. Producción actual, biomasa y composición específica del fitoplancton de la Bahía de Valparaíso en 1979. Revista de Biología Marina 18(2):129157.

Avendaño M. 1993. Données sur la biologie de Argopecten purpuratus (Lamarck, 1819), Mollusque Bivalve du Chili. Thèse doctorat, Universidad de Bretagne Occidentale, Brest, $167 \mathrm{pp}$.

Avendaño M \& M Cantillánez. 1996. Efecto de la pesca clandestina sobre Argopecten purpuratus (Lamarck, 1819) en el banco de La Rinconada, II Región. Ciencia y Tecnología del Mar 19: 57-65.

Avendaño M \& M Cantillánez. 1997. Necesidad de crear una reserva marina en el banco de ostiones de La Rinconada - II Región. Estudios Oceanológicos 16: 109-113.

Avendaño M \& M Cantillánez. 2005. Growth and population structure of Argopecten purpuratus at La Rinconada marine reserve, Antofagasta, Chile. Ciencias Marinas 31(3): 491503.
Avendaño M \& M Cantillánez. 2008. Aspectos biológicos y poblacionales de Argopecten purpuratus (Lamarck, 1819) en la Reserva Marina la Rinconada: contribución para su manejo. En: Lovatelli A, A Farías \& I Uriarte (eds). Estado actual del cultivo y manejo de moluscos bivalvos y su proyección futura: Factores que afectan su sustentabilidad en América Latina. Actas de Pesca de la FAO 12: 249-266.

Avendaño M \& M Le Pennec. 1996. Contribución al conocimiento reproductivo de Argopecten purpuratus (Lamarck, 1819), en dos poblaciones de la II Región - Chile. Estudios Oceanológicos 15: 1-10

Avendaño M \& M Le Pennec. 1997. Intraspecific variation in gametogenesis in two populations of the Chilean Molluscan Bivalve, Argopecten purpuratus (Lamarck). Aquaculture Research 28: 175-182

Avendaño M, M Le Pennec \& M Cantillánez. 2001. Anormalidades en larvas de Argopecten purpuratus (Lamarck, 1819) (Mollusca, Pectinidae); una causal de los problemas en la producción artificial de semilla. Estudios Oceanológicos 20: 33-42.

Avendaño M, M Cantillánez \& J Peña. 2006. Effect of immersion time of cultch on spatfall of the scallop Argopecten purpuratus (Lamarck 1819) in the Marine Reserve at La Rinconada, Antofagasta, Chile. Aquaculture International 14: 267-283.

Avendaño M, M Cantillánez, M Le Pennec \& G Thouzeau. 2008a. Reproductive and larval cycle of the scallop Argopecten purpuratus (Ostreoida: Pectinidae), during El Niño-La Niña events and normal weather conditions in Antofagasta, Chile. Revista de Biología Tropical 56(1): 121132

Avendaño M, M Cantillánez \& G Thouzeau. 2008b. Effects of depth on the growth and survival of Argopecten purpuratus spat (Lamarck, 1819) in artificial collectors in northern Chile. Aquaculture International 16: 377-391.

Bojórquez-Verástica G. 1997. Reclutamiento, crecimiento y supervivencia de Argopecten circularis, (Sowerby, 1935), Argopecten ventricosus, (Sowerby II, 1842), en Bahía Concepción, BCS. Tesis de Licenciatura, Universidad Autónoma de Baja California Sur, La Paz, 72 pp.

Boucher J. 1985. Caractéristiques dynamiques du cycle vital de la coquille Saint-Jacques (Pecten maximus): hypothèses sur les stades critiques pour le recrutement. Rapports et ProcèsVerbaux des Réunions / Conseil Permanent International Pour l'Exploration de la Mer, ICES CM K23 : 1-10.

Boucher J \& S Fifas. 1995. Dynamique de la population de coquilles St-Jacques (Pecten maximus) de la rade de Brest, hier était-il différent d' aujourd' hui? Actes de colloques, 3ièmes Rencontres Scientifiques Internationales 2: 2-12. Programme Rade de Brest.

Caddy JF. 1989. A perspective on the population dymanics and assessment of scallop fishery with special reference to sea scallop, Placopecten magellanicus Gmelin. In: Caddy JF (ed). Marine invertebrate fisheries: Their assessment \& management 25: 559-589. John Wiley and Sons, New York. 
Cantillánez M. 2000. Reproduction, vie larvaire et prérecrutement du Pectinidae Argopecten purpuratus (Lamarck, 1819) dans la baie d'Antofagasta (Chili). Thèse doctorat, Université de Bretagne Occidentale, Brest, 168 pp.

Cantillánez M, G Thouzeau \& M Avendaño. 2001. Reproductive cycle in Argopecten purpuratus during El Niño and la Niña conditions: a case study in the Rinconada Bay (Chile). In: Illanes JE \& E von Brand (eds). Book of Abstracts 13th International Pectinid Workshop, Coquimbo, Chile. pp. 86-88. Universidad Católica del Norte, Coquimbo.

Cantillánez M, M Avendaño \& G Thouzeau. 2005. Reproductive cycle of Argopecten purpuratus (Bivalvia: Pectinidae) in La Rinconada Marine Reserve (Antofagasta, Chile): response to environmental effects of El Niño and $\mathrm{La}$ Niña. Aquaculture 246: 181-195.

Cantillánez M, G Thouzeau \& M Avendaño. 2007. Improving Argopecten purpuratus (Bivalvia: Pectinidae) culture in La Rinconada marine reserve (Antofagasta, Chile): Results from the study of larval and post-larval stages in relation to environmental forcing. Aquaculture 272: 423-443.

Carlssona L \& F Berkesb. 2005. Co-management: concepts and methodological implications. Journal of Environmental Management 75: 65-76.

Dickie LM. 1995. Fluctuation in abundance of the giant scallop, Placopecten magellanicus (Gmelin) in the Digby area of the Bay of Fundy. Journal of Fisheries Research, Board of Canada 12(6): 797-857.

Escribano R, L Rodríguez \& C Irribarren. 1995. Temporal variability of sea temperature in Bay of Antofagasta, northern Chile (1991-1995). Estudios Oceanológicos 14: 39-47.

Gwyther D \& D Burgess. 1987. Abundance of scallops in Port Phillip Bay and prediction of yields for the 1987 season. Marine Science Laboratory, Technique Report 64: 1-12. Department of Conservation Forests and Lands, Victoria.

Jones PJS. 2009. Equity, justice and power issues raised by no-take marine protected area proposals. Marine Policy 33: 759-765.

MacDonald PDM \& TJ Pitcher. 1979. Age-groups from sizefrequency data: a versatile and efficient method of analyzing distribution mixtures. Journal of the Fisheries Research Board of Canada 36: 987-1001.

Maeda-Martínez AN, MT Sicard, L Carvalho, SE LluchCota \& DB Lluch-Cota. 2001. Las poblaciones de almeja catarina Argopecten ventricosus en el Centro de actividad biológica de Bahía Magdalena, México. En: Lluch-Belda D, J Elorduy-Garay, SE Lluch-Cota \& G Ponce-Diaz (eds). Centros de actividad biológica del Pacífico Mexicano, pp. 219-228. Editorial CIBNOR, México.

Marín V \& L Delgado. 1997. Nueva estrategia para un desarrollo sustentable: Manejo Ecosistémico de los Recursos Naturales. Ambiente y Desarrollo, Chile 13(2): 70-76.

Mason J. 1983. Scallop and queen fisheries in the British Isles, 147 pp. Fishing News Books, London.
Masso-Rojas JA. 1996. Pesquería de la almeja catarina. En: Casas-Valdéz M \& G Ponce-Díaz (eds). Estudio del potencial pesquero y acuícola de Baja California Sur 1: 71-85, CIBNOR, La Paz.

Meffe GK \& CR Carroll. 1994. Principles of conservation biology, 600 pp. Sinauer Associates, Sunderland.

Morales H \& L Gezan. 1986. La modernización de las pesquerías chilenas: impactos sociales y ecológicos. Ambiente y Desarrollo 2(2): 33-46.

Murawski S, R Brown, H Lai, P Rago \& L Hendrickson. 2000. Large-scale closed areas as a fishery-management tool in temperate marine systems: the Georges Bank experience. Bulletin of Marine Science 66(3): 775-798.

Narvarte M, E Félix-Pico \& L Ysla-Chee. 2001. Asentamiento larvario de pectínidos, en colectores artificiales. En: Maeda-Martínez AN (ed). Los moluscos pectínidos de Iberoamerica: Ciencia y acuicultura, pp. 173-192. Editorial Limusa, México.

Nursey-Bray M \& P Rist. 2009. Co-management and protected area management: Achieving effective management of a contested site, lessons from the Great Barrier Reef World Heritage Area (GBRWHA). Marine Policy 33: 118-127.

Orensanz J, A Parma \& O Iribarne. 1991a. Population dynamics and management of natural stocks. In: Shumway SE (ed). Scallops: Biology, ecology, and aquaculture, pp. 625713. Elsevier, New York.

Orensanz J, M Pascual \& M Fernandez. 1991b. Scallops in Argentina. In: Shumway SE (ed). Scallops: Biology, ecology, and aquaculture, pp. 991- 996. Elsevier, New York.

Ortiz M, M Avendaño, L Campos \& F Berrios. 2009. Spatial and mass balanced trophic models of La Rinconada Marine Reserve (SE Pacific coast), a protected benthic ecosystem: Management strategy assessment. Ecological Modelling 220: 3413-3423.

Ortiz M, M Avendaño, M Cantillánez, F Berrios \& L Campos. 2010. Trophic mass balanced models and dynamic simulations of benthic communities from La Rinconada Marine Reserve off northern Chile: network properties and multispecies harvest scenario assessment. Aquatic Conservation: Marine and Freshwater Ecosystems 20(1): 5873.

Paulet YM, F Bekhadra, N Devauchelle, A DonvaL \& G Dorange. 1997. Cycles Saisonniers, Reproduction et Qualité des Ovocytes Chez Pecten maximus en Rade de Brests. Annales de l'Institut Océanographique 73(1): 101-112.

Pomeroy RS, JE Parks \& LM Watson. 2006. Cómo evaluar una AMP. Manual de indicadores naturales y sociales para evaluar la efectividad de la gestión de Áreas Marinas Protegidas, 216 pp. UICN, Gland.

Rodriguez L. 1987. Observaciones sobre fitoplancton y temperatura superficial en la Bahía San Jorge, Antofagasta, Chile. Revista de Biología Marina 23(1): 1-29. 
Rodriguez L \& R Escribano. 1996. Bahía Antofagasta y Bahía de Mejillones del Sur: observaciones de la temperatura, penetración de la luz, biomasa y composición fitoplanctónica. Estudios Oceanológicos 15: 75-85

Sause B, D Gwyther \& D Burgess. 1987. Larval settlement, juvenile growth and potential use of spatfall indices to predict recruitment of the scallop Pecten alba Tate in Port Phillip Bay, Victoria, Australia. Fisheries Research 6: 81-92.

Sinclair M, R Mohn, G Probert \& D Roddick. 1985. Considerations for the effective management of Atlantic scallops. Canadian Technical Report of Fisheries and Aquatic Sciences 1382: 1-97.

Stotz WB. 2000. When aquaculture restores and replaces a overfished stock: is the conservation of the species assured? The case of the scallop Argopecten purpuratus (Lamarck, 1819) in northern Chile. Aquaculture International 8: 237 247.

Stotz WB \& J Mendo. 2001. Pesquería, repoblamiento y manejo de bancos naturales de pectínidos en Iberoamérica: su interacción con la acuicultura. En: Maeda-Martínez AN (ed) Los moluscos pectínidos de Iberoamerica: Ciencia y acuicultura, pp. 357-374. Editorial Limusa, México.

Thompson S. 1992. Sampling, 345 pp. Wiley-Intercience, New York.

Thouzeau G. 1991a. Experimental collection of postlarvae of Pecten maximus (L.) and other benthic macrofaunal species in the Bay of Saint-Brieuc, France. I- Settlement patterns and biotic interaction among the species collected. Journal of Experimental Marine Biology and Ecology 148: 159-179.
Thouzeau G. 1991b. Experimental collection of postlarvae of Pecten maximus (L.) and other benthic macrofaunal species in the Bay of Saint-Brieuc, France. II- Reproduction patterns and postlarval growth of five mollusk species. Journal of Experimental Marine Biology and Ecology 148: 181-200.

Thouzeau G. 1991c. Déterminisme du pré-recrutement de Pecten maximus (L.) en baie de Saint-Brieuc: processus régulateurs de l'abondance, de la survie et de la croissance des post-larves et juvéniles. Aquatic Living Resources 4: 77 99.

Thouzeau G \& D Lehay. 1988. Variabilité spatio-temporelle de la distribution, de la croissance et de la survie des juveniles de Pecten maximus (L.) issus des pontes de 1985 en la baie de Saint-Brieuc. Oceanologica Acta 11(13): 267-284.

Thouzeau G, M Avendaño, L Chauvaud, M Cantillanez \& O Strand. 2001. Enviromental monitoring: an absolute necessity for scallop aquaculture in coastal ecosystems facing antropogenic perturbation. In: Illanes JE \& E Von Brand (eds). Book of Abstracts 13th International Pectinid Workshop. Coquimbo, Chile, pp. 66-68. Universidad Católica del Norte, Coquimbo.

Wroblewski J, T Bell, A Copeland, E Edinger, Ch Yu Feng, J Saxby, D Schneider \& J Simms. 2009. Toward a sustainable Iceland scallop fishery in Gilbert Bay, a marine protected area in the eastern Canada coastal zone. Journal of Cleaner Production 17: 424 - 430. 